\title{
OPEN Author Correction: Genomic prediction and GWAS of yield, quality and disease-related traits in spring barley and winter wheat
}

Hsin-Yuan Tsai, Luc L. Janss, Jeppe R. Andersen, Jihad Orabi, Jens D. Jensen, Ahmed Jahoor \& Just Jensen

Correction to: Scientific Reports https://doi.org/10.1038/s41598-020-60203-2, published online 25 February 2020

This Article contains errors in Tables 4 and 5, where in the "Trait" column in Table 4 and the "Trait Combination" column in Table 5, the row title "Straw breaking" for the barley species was incorrectly given as "Starch".

The correct Tables 4 and 5 appear below.

\begin{tabular}{|c|c|c|c|c|c|c|}
\hline & Trait & Marker & Chromosome & Position(cM) & P-value & $\begin{array}{l}\text { Genomic } \\
\text { Effect* }\end{array}$ \\
\hline \multirow{16}{*}{ Barley } & \multirow{8}{*}{ Powdery Mildew } & SNP1 & $4 \mathrm{H}$ & 100.68 & $1.09 \mathrm{E}-20$ & 10.70 \\
\hline & & SNP2 & $4 \mathrm{H}$ & 101.28 & $1.72 \mathrm{E}-19$ & 10.30 \\
\hline & & SNP3 & $4 \mathrm{H}$ & 99.68 & $2.73 \mathrm{E}-19$ & 10.00 \\
\hline & & SNP4 & $4 \mathrm{H}$ & 102.38 & $9.75 \mathrm{E}-19$ & 10.20 \\
\hline & & SNP5 & $4 \mathrm{H}$ & 101.68 & $1.23 \mathrm{E}-13$ & 6.70 \\
\hline & & SNP6 & $4 \mathrm{H}$ & 102.08 & $1.68 \mathrm{E}-11$ & 5.55 \\
\hline & & SNP7 & $4 \mathrm{H}$ & 102.18 & $1.95 \mathrm{E}-11$ & 5.53 \\
\hline & & SNP8 & Unknown & Unknown & $3.89 \mathrm{E}-10$ & 5.03 \\
\hline & \multirow{2}{*}{ Ramularia } & SNP6 & $4 \mathrm{H}$ & 102.08 & $2.69 \mathrm{E}-07$ & 7.03 \\
\hline & & SNP7 SNP9 & 4H Unknown & 102.18 Unknown & $2.92 \mathrm{E}-07$ 4.01E-07 & 7.006 .11 \\
\hline & \multirow{4}{*}{ Yield } & SNP10 & $4 \mathrm{H}$ & 88.84 & $5.85 \mathrm{E}-07$ & 0.75 \\
\hline & & SNP11 & $4 \mathrm{H}$ & 88.84 & $5.85 \mathrm{E}-07$ & 0.75 \\
\hline & & SNP12 & $4 \mathrm{H}$ & 88.84 & $5.85 \mathrm{E}-07$ & 0.75 \\
\hline & & SNP13 & $4 \mathrm{H}$ & 88.64 & $1.76 \mathrm{E}-06$ & 0.68 \\
\hline & Straw breaking & SNP14 & $4 \mathrm{H}$ & 32.24 & $6.19 \mathrm{E}-08$ & 18.60 \\
\hline & \begin{tabular}{|l|} 
Lodging \\
\end{tabular} & SNP14 & $4 \mathrm{H}$ & 32.24 & \begin{tabular}{|l}
$4.44 \mathrm{E}-09$ \\
\end{tabular} & 12.70 \\
\hline \multirow{4}{*}{ Wheat } & \multirow{3}{*}{ Moisture } & SNP15 & $6 \mathrm{~A}$ & 40.8 & $1.22 \mathrm{E}-06$ & 1.34 \\
\hline & & SNP16 & $1 \mathrm{~B}$ & 201.25 & $2.16 \mathrm{E}-06$ & 1.44 \\
\hline & & SNP17 & $6 \mathrm{~A}$ & 30.04 & $3.13 \mathrm{E}-06$ & 1.23 \\
\hline & Starch Content & SNP18 & $5 B$ & 222.57 & 4.17E-06 & 4.09 \\
\hline
\end{tabular}

Table 4. Genome-wide significant markers in univariate GWAS in spring barley and winter wheat $(\mathrm{P}<0.05)$. *The genomic effect value is multiplied by $10^{3}$. 


\begin{tabular}{|c|c|c|c|c|c|}
\hline & Trait Combination & Marker & Chromosome & Position(cM) & P-value \\
\hline \multirow{19}{*}{ Barley } & \multirow{14}{*}{$\begin{array}{l}\text { Mildew } \\
\text { Ramularia }\end{array}$} & SNP1 & $4 \mathrm{H}$ & 100.68 & $2.18 \mathrm{E}-20$ \\
\hline & & SNP2 & $4 \mathrm{H}$ & 101.28 & $3.44 \mathrm{E}-19$ \\
\hline & & SNP3 & $4 \mathrm{H}$ & 99.68 & $5.46 \mathrm{E}-19$ \\
\hline & & SNP4 & $4 \mathrm{H}$ & 102.38 & $1.95 \mathrm{E}-18$ \\
\hline & & SNP5 & $4 \mathrm{H}$ & 101.68 & $2.45 \mathrm{E}-13$ \\
\hline & & SNP6 & $4 \mathrm{H}$ & 102.08 & $3.36 \mathrm{E}-11$ \\
\hline & & SNP7 & $4 \mathrm{H}$ & 102.18 & $3.91 \mathrm{E}-11$ \\
\hline & & SNP8 & Unknown & Unknown & $7.78 \mathrm{E}-10$ \\
\hline & & SNP19 & $4 \mathrm{H}$ & 97.38 & $2.59 \mathrm{E}-08$ \\
\hline & & SNP20 & $4 \mathrm{H}$ & 98.65 & $6.51 \mathrm{E}-07$ \\
\hline & & SNP9 & Unknown & Unknown & $8.01 \mathrm{E}-07$ \\
\hline & & SNP21 & $4 \mathrm{H}$ & 97.29 & 6.64E-06 \\
\hline & & SNP22 & $4 \mathrm{H}$ & 97.19 & $6.70 \mathrm{E}-06$ \\
\hline & & SNP23 & $4 \mathrm{H}$ & 97.19 & $9.18 \mathrm{E}-06$ \\
\hline & \multirow{2}{*}{ Yield } & SNP14 & $4 \mathrm{H}$ & 32.24 & $1.27 \mathrm{E}-08$ \\
\hline & & SNP10 & $4 \mathrm{H}$ & 88.84 & $1.68 \mathrm{E}-06$ \\
\hline & \multirow{2}{*}{ Straw breaking } & SNP11 & $4 \mathrm{H}$ & 88.84 & $1.68 \mathrm{E}-06$ \\
\hline & & SNP12 & $4 \mathrm{H}$ & 88.84 & $1.68 \mathrm{E}-06$ \\
\hline & Lodging & SNP13 & $4 \mathrm{H}$ & 88.64 & $5.04 \mathrm{E}-06$ \\
\hline Wheat & $\begin{array}{l}\text { Moisture } \\
\text { Starch } \\
\text { Yield }\end{array}$ & SNP15 & $6 \mathrm{~A}$ & 40.8 & 3.57E-06 \\
\hline
\end{tabular}

Table 5. Genome-wide significant markers in multivariate GWAS in spring barley and winter wheat $(P<0.05)$. *The markers were not identified in univariate GWAS (cf. Table 4).

(i) Open Access This article is licensed under a Creative Commons Attribution 4.0 International License, which permits use, sharing, adaptation, distribution and reproduction in any medium or format, as long as you give appropriate credit to the original author(s) and the source, provide a link to the Creative Commons license, and indicate if changes were made. The images or other third party material in this article are included in the article's Creative Commons license, unless indicated otherwise in a credit line to the material. If material is not included in the article's Creative Commons license and your intended use is not permitted by statutory regulation or exceeds the permitted use, you will need to obtain permission directly from the copyright holder. To view a copy of this license, visit http://creativecommons.org/licenses/by/4.0/.

(c) The Author(s) 2020 